INTERNATIONAL JOURNAL OF SCIENTIFIC RESEARCH

\title{
PLEOMORPHIC ADENOMA IN AN UNUSUAL SITE
}

\section{General Surgery}

Dr Ruban Kumar J* Junior Resident in Surgery, Saveetha Medical College,SIMATS. *Corresponding Author

Dr Shobana M Junior Resident in Surgery, Saveetha Medical College,SIMATS.

Dr Dinesh K B $\quad$ Senior Resident in Surgery, Saveetha Medical College,SIMATS.

Dr Shruthi kamal V Professor of Surgery, Saveetha Medical College,SIMATS.

\section{ABSTRACT}

Pleomorphic adenoma is also called as mixed parotid tumour, which is a benign neoplasm of the salivary gland, occurring more frequently in major salivary gland. It occurs infrequently in minor salivary glands. It can involve the palate and lips as well.

The aim of this paper is to report the case of a 24 year old male, admitted in Saveetha medical college and hospital, with complaints of swelling in the upper lip for 6 months, which was successfully diagnosed as pleomorphic adenoma and treated with surgical excision.

\section{KEYWORDS}

\section{INTRODUCTION}

The pleomorphic adenoma, or benign mixed tumour, is the most common salivary gland neoplasm, accounting for $60-65 \%$ of all major and minor salivary gland tumours. It consists $53-77 \%$ of parotid tumours, $44-68 \%$ of submandibular tumours, and $38-43 \%$ of minor salivary gland tumours. The palate followed by the upper lip appears to be the most affected intraoral site of the minor salivary glands pleomorphic adenoma. Eighty percent of minor salivary gland tumours in lip are benign. Clinically, the lesion occurs as a painless, slow-growing, dome-shaped mass with a smooth surface. The microscopic examination shows a mixture of glandular epithelial and myoepithelial cells within a mesenchyme-like background. The aetiology of the tumour is unknown but irradiation of the head and neck increases the risk of developing this tumour. This paper elaborates on the diagnosis and management of a upper lip pleomorphic adenoma.

\section{CASE REPORT}

A 24-year-old male patient presented with complaints of a painless swelling in the central portion of his upper lip for past 6 months. He did not have pain or discharge associated with the swelling. The patient had no difficulty in mouth opening and functional disability. There was no history of similar swelling elsewhere in the body and no relevant medical and dental history. On clinical examination there was $3 \times 2 \mathrm{~cm}$ firm, non-tender, nodular, circumscribed lesion present in the area of philtrum, obliterating its depression just adjacent to the collumelarphiltral junction of the upper lip. Swelling was non-compressible, nonreducible and non-pulsatile. There was no regional lymphadenopathy. Based on the presence of a slowly growing, painless mass located in the upper lip in a young adult, a benign salivary gland tumour was considered as the provisional diagnosis. The differential diagnosis of a lipoma was considered.

Excision was performed under local anaesthesia and the specimen was sent for histopathological examination. The post operative period was uneventful.

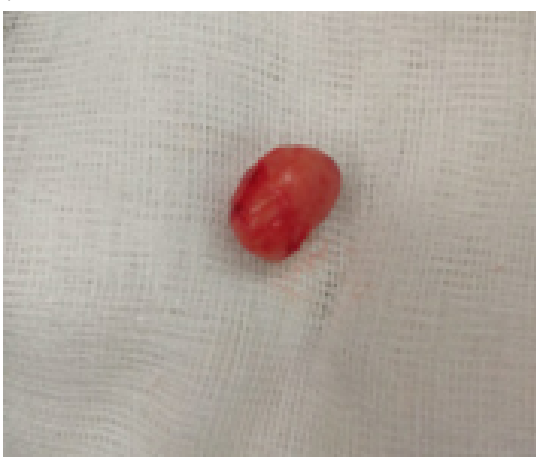

Fig. 1, showing postoperative excised specimen. $2 \times 3 \times 3 \mathrm{~cm}$ globular soft tissue mass.

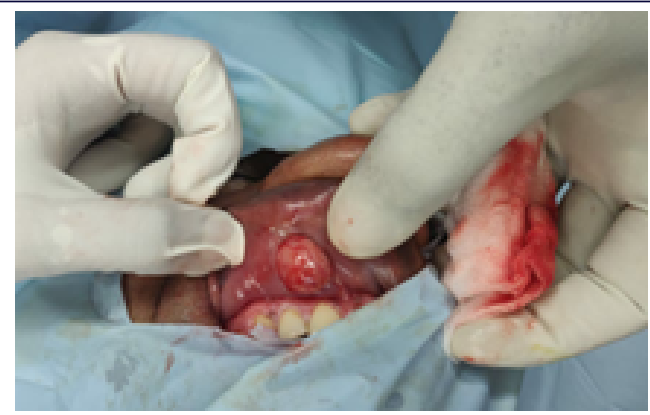

Fig. 2, showing intra-operative image of soft tissue mass in the upper lip.

A single grey white globular soft tissue mass measuring $3 \times 2 \times 2 \mathrm{~cm}$ was taken for histopathological examination. Section showed focal salivary gland structure with a benign neoplasm composed of sheets and cords of benign epithelial cells with abundant myxoid and chondroid areas. No atypia seen.

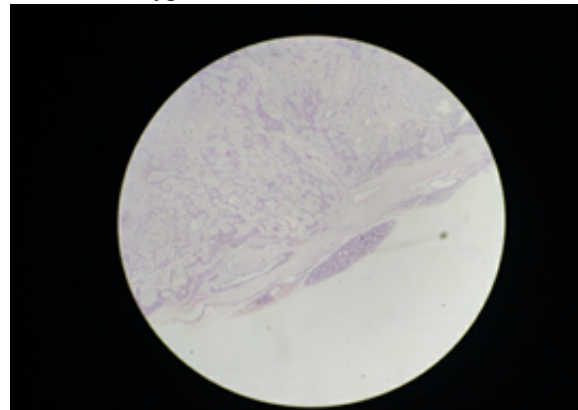

Fig. 3,4, showing sheets and cords of benign epithelial cells with abundant myxoid and chondroid areas.

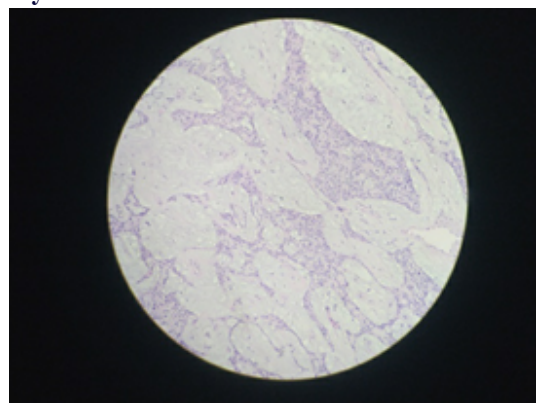

Fig. 4. The above mentioned features were suggestive of pleomorphic adenoma. After 6 months of regular follow-up no loco regional recurrence was observed. 
The minor salivary glands are widely dispersed in the upper respiratory tract, including the lip, palate, pharynx, larynx and nasopharynx (4). In head and neck neoplasm, salivary gland tumours are rare and constitute $3-5 \%$ (2). In $10-15 \%$ cases it affects minor salivary gland (2). Lips are the integral structure of lower third face that acts as a muscular sphincter guarding the opening of the oral cavity (1). It is made up of both epithelial and mesenchymal connective tissue components that is skin, adipose tissue, orbicularis oris muscle, minor salivary glands, blood vessels and nerves (1). Among both the lips, upper lip is more prone to developmental anomalies such as cleft lip whereas lower lip is more affected by pathological conditions like mucocele and other neoplastic conditions (1). Bernier found that the peak incidence of pleomorphic adenoma of the lips was in the third and fourth decades, with an average age of 33.2 years (3). Owens and calcaterra found $90 \%$ of the upper lip tumours to be benign (2). Eveson and cawson found $75 \%$ of the upper lip tumours as benign (3). Kroll and Hick reviewed 4042 cases of pleomorphic adenomas of the salivary glands. Of these, 445 originated in the minor salivary glands, only $16.9 \%$ were located in the upper lip (4). Daley reviewed the clinical differential diagnosis of a swelling of the upper lip, listing mucocele, fibroma, lipoma, mucus retention cyst, sialolith, phlebolith and salivary gland neoplasm as possibilities (1).

Among benign salivary gland pathologies, canalicular adenoma almost always occurs in the upper lip, most often at the midline (1). Pleomorphic adenoma is the second most common benign tumour of the upper lip (1). This tumour has been referred to by a great variety of names through the years- Enclavoma, Branchioma, Endothelioma, Enchondroma, but the term pleomorphic adenoma suggested by WILLS characterizes closely the unusual histological pattern of the lesion (4). As epithelial and mesenchymal components are present, it is known as benign mixed tumour (2).

The most frequent site of pleomorphic adenoma of minor salivary gland is hard and soft palate (42-68\%), followed by upper lip (10\%) and rarely in tongue, floor of mouth, and retromolar area (2). Usually lesions in upper lip are benign, whereas malignant lesions tend to occur in lower lip (2). Pleomorphic adenoma usually presents as slow growing painless nodular firm mass which is freely mobile, so as in ur case (2). The malignant transformation may occur in $5 \%$ of cases (2).

As pleomorphic adenoma is rare in this location, clinical examination and histopathology study is required for definitive diagnosis (1). The tumour is usually encapsulated, so surgical excision with adequate surrounding tissue margins is treatment of choice (1). Histologically, it is typically well circumscribed encapsulated tumour but lack of complete encapsulation is more common for minor salivary gland tumours (4). Histologically, the tumour mainly comprises of epithelial, myoepithelial cells and mesenchyme like components. Epithelium usually forms sheets or duct like structures. Dardick has described the neoplastic myoepithelial cells in pleomorphic adenoma as "swarm of bees" enclosing ductal structures (4). Recurrence is rare in minor salivary gland tumours (1). The reason for recurrence is rupture of tumour capsule or incomplete excision during the procedure and leaving behind live residual tumour cells (2). The recurrence of pleomorphic adenoma is $2-44 \%$ (2). Pleomorphic adenoma ha excellent prognosis with a cure rate of $95 \%(2)$.

\section{CONCLUSION}

This case report describes a rare unusual lesion found in a 24-year-old male, which was diagnosed as pleomorphic adenoma of the minor salivary glands in the upper lip. Pleomorphic adenoma consists of cells with epithelial and mesenchymal differentiation. The treatment of choice for pleomorphic adenoma is surgical removal with safety margins, to prevent the recurrence. Periodic follow-up is must due to the important relapse potential.

\section{REFERENCES -}

1) Pleomorphic adenoma involving minor salivary glands of upper lip: A rare phenomenon. Singh akileshkumar, Kumar Naresh, Sharma parikshit, Singh Shreya.

2) Bhatia, JSS. Pleomorphic adenoma of upper lip: A rare case presentation. Indian J otolaryngol head neck surg 71, 755-758 (2019).

3) Natl J Maxillofac surg. 2011 jul-dec; 2(2):219-221. Iqbal Ali, Anup Kumar Gupta, Saurabh Singh.

4) Pleomorphic adenoma: An unusual presentation on upper lip - A case report. Thomas Deena C, Nair Vivek V, Thomas sunila. Journal of indian academy of oral medicine and radiology 29(3), 217-219(2017). 